Ophthalmologe 2015 · 112:312-312

DOI 10.1007/s00347-014-3159-0

Online publiziert: 27. Februar 2015

(c) Springer-Verlag Berlin Heidelberg 2015

T. Klink

Julius-Maximilians-Universität Würzburg

\title{
Kanaloplastik und andere nicht penetrierende Glaukomoperationen
}

beschriebenen Viskokanalostomie entwickelt hat. Sie zielt auf eine Druckregulation durch die Wiederherstellung des natürlichen Abflussweges. Auf die Ausbildung eines Sickerkissens wird bewusst verzichtet, um die damit einhergehenden Komplikationen wie Vernarbung, Blebitis und Endophthalmitis zu vermeiden. Der Wirkmechanismus der Kanaloplastik ist noch nicht sicher geklärt. Er scheint zum einen auf einem Pilocarpineffekt durch den in den Schlemm-Kanal eingezogenen Faden zu beruhen, zum anderen erzeugt die Dehnung des Trabekelmaschenwerks sicher Mikrorupturen, die ähnlich einer Trabekulotomie einzustufen sind. Inwieweit eine Vernarbung im Bereich des Trabekelwerks langfristig die Funktion der Operation beeinträchtigt, ist noch nicht bekannt. Die Möglichkeiten einer intraoperativen Einschätzung der Funktion des Abflusssystems, insbesondere des peripheren Anteils der Kollektorkanäle und Kammerwasservenen werden in dem Beitrag von Herrn PD Grieshaber dargestellt. Betrachtet man den drucksenkenden Effekt der Kanaloplastik, so scheint dieser etwas geringer im Vergleich zur Trabekulektomie zu sein. Die Zahl der Medikamente, die postoperativ zur Augeninnendrucksenkung benötigt werden, liegt höher verglichen mit der penetrierenden Chirurgie. Das komplikationsärmere Verfahren ist im direkten Vergleich die Kanaloplastik. Auch die Zahl der postoperativen Interventionen ist nach Kanaloplastik geringer. Gerade für Patienten mit erhöhtem Komplikationsrisiko, weit fortgeschrittenen Gesichtsfelddefekten oder eingeschränkter Möglichkeit der postoperativen Nachsorge ist die Kanaloplas- tik sicher eine Alternative zur Trabekulektomie. Im Beitrag von Herrn Prof. Körber wird die Möglichkeit, die Kanaloplastik auch als Zweiteingriff nach Trabekulektomie durchzuführen, aufgegriffen. Das individuelle Therapiespektrum für den $\mathrm{Pa}$ tienten kann damit interessant erweitert werden.

Die nicht penetrierenden Operationstechniken haben mittlerweile ihren festen Platz in der chirurgischen Therapie der Glaukome und stellen einen wichtigen Zwischenschritt in der Entwicklung von komplikationsärmeren Eingriffen mit hoher Effektivität dar. Zudem erweitern sie, wie in vielen anderen Bereichen der Ophthalmologie, die therapeutischen Optionen für unsere Patienten hin zu einer individualisierten, auf den Einzelnen zugeschnittenen Behandlung.

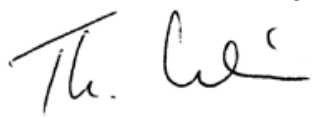

Thomas Klink

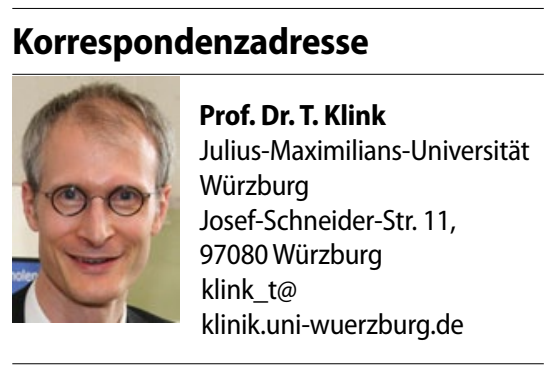

Einhaltung ethischer Richtlinien

Interessenkonflikt. T. Klink gibt an, dass kein Interessenkonflikt besteht.

Dieser Beitrag beinhaltet keine Studien an Menschen oder Tieren. 